\title{
Una revisión de los métodos de Deep Learning aplicados a la detección automatizada de la retinopatía diabética
}

\author{
José Cuello Navarro, Carlos Barraza Peña \& José Escorcia-Gutiérrez \\ Ingeniería Electrónica y Telecomunicaciones, Universidad Autónoma del Caribe, Barranquilla, Colombia \\ josecnav27@gmail.com, carlosbarraz.05521@gmail.com,jose.escorcia23@gmail.com
}

Recibido: Agosto 23, 2020.

Recibido en su versión corregida: Octubre 05, 2020.

Aceptación: Octubre 15, 2020.

Cómo citar: Cuello Navarro, J., Barraza Peña, C. \& Escorcia-Gutierrez, J. (2020). Una revisión de los métodos de deep learning aplicados a la detección automatizada de la retinopatía diabética. Revista Sextante, 23, pp. 12 - 27, 2020.

\section{Resumen}

La retinopatía diabética $(\mathrm{RD})$ es una enfermedad ocular caracterizada por cambios anormales a nivel de la retina, que se manifiesta con gran frecuencia en personas que padecen diabetes mellitus. El debido seguimiento y tratamiento puede evitar que la enfermedad afecte gravemente al paciente, sin embargo, debido a la necesidad de contar con profesional especializado y lo costoso del proceso, puede resultar difícil realizar diagnósticos periódicamente, corriendo el riesgo de presentar ceguera. Para contribuir a la solución de dicho problema, se han visto en los últimos años trabajos que emplean tecnología de inteligencia artificial, más precisamente aplicando técnicas de Deep Learning (DL) para el reconocimiento automatizado de los síntomas de la RD presentes en la retina. En este artículo se plantean las bases para la detección de la RD, así como las técnicas DL utilizadas por diversos investigadores para su diagnóstico automatizado y el desempeño de dichos sistemas en este ámbito.

Palabras claves: Clasificación; Diagnóstico; Procesamiento de imágenes médicas; Redes neuronales convolucionales; Retinopatía diabética.

\section{A survey on deep learning for automated detection of diabetic retinopathy}

\begin{abstract}
Diabetic Retinopathy (DR) is an eye disease characterized by abnormal changes at the level of the retina that is very common in people with Diabetes Mellitus. The proper monitoring and treatment can prevent the disease from seriously affecting the patient, however, due to the need for specialized professionals and the cost of the process, it can be difficult to make periodic diagnoses, running the risk of blindness. In order to contribute to the solution of this 1ystems1, in recent years there have been 1yste that use artificial intelligence technology, more precisely applying Deep Learning (DL) techniques for the automated recognition of the DR symptoms present in the retina. In this article, the bases for the detection of DR are presented, as well as the DL techniques used by various researchers for automated diagnosis and the performance of these 1ystems in this field.
\end{abstract}

Keywords: Convolutional neural networks; Diabetic retinopathy; Diagnosis; Image classification; Medical image análisis. 


\section{Introducción}

La visión es uno de los sentidos más indispensables que tienen los seres humanos para la realización de actividades en el día a día. El ojo es el órgano sensorial responsable de nuestra vista y se compone de varias partes como la pupila, el cristalino, el iris, la córnea, la retina y otras. Ahora bien, la retina es la parte del ojo en donde se manifiestan la mayoría de los síntomas producidos por enfermedades relacionadas a la visión, el globo ocular y el sistema circulatorio. (Mittal \& Rajam, 2020).

Una de estas enfermedades es la retinopatía diabética, la cual según Scanlon (2019), se refiere a la patología de los capilares, arteriolas y vénulas de la retina, y los efectos posteriores de fuga u oclusión de los vasos pequeños. Los cambios que ocurren dentro de los capilares de la retina incluyen engrosamiento de la membrana basal, disfunción de las células epiteliales (pérdida de las uniones epiteliales estrechas), pérdida de células endoteliales, aumento de la permeabilidad capilar, debilitamiento capilar, muerte de las células del músculo liso, oclusión capilar y formación de micro aneurismas.

Pero aquí el problema fundamental de la retinopatía diabética lo explica Asiri et al. (2019) cuando menciona que esta enfermedad se vuelve incurable en etapas avanzadas, por lo que el diagnóstico precoz es importante. Sin embargo, esto implica una dificultad notable en el sistema sanitario debido a la gran cantidad de pacientes potenciales y al reducido número de técnicos experimentados. Esta situación ha llevado al desarrollo de diversos sistemas de diagnóstico automático para ayudar en el diagnóstico precoz de la $\mathrm{RD}$, los cuales se soportan en técnicas y métodos obtenibles gracias a los avances tecnológicos que ha logrado la ciencia de la inteligencia artificial.

La inteligencia artificial, y en especial las redes enfocadas al aprendizaje profundo (DL, por sus siglas en inglés), son modelos computacionales que intentan emular la función que ejecuta el cerebro humano. Estás tienen la capacidad de poder aprender mediante un entrenamiento y se han convertido en una herramienta muy reconocida por su amplia variedad y diversidad de aplicación en muchas ramas de la ciencia. (Rodríguez, 2019).
Según Perdomo \& González (2019), en el mundo de la medicina la aplicación de métodos de aprendizaje profundo se ha visto principalmente en la rama de la oftalmología con el análisis de imágenes de fondo de ojo y en tomografías de coherencia óptica. El panorama según estos investigadores indica que dichos métodos han logrado un rendimiento de alto impacto en la detección de enfermedades oculares, tales como la retinopatía diabética (RD), el glaucoma, la degeneración macular diabética y la degeneración relacionada con la edad.

Por otro lado, varios investigadores y competencias realizadas a nivel mundial han desarrollado trabajos y colocado, a la disposición de toda la comunidad científica e investigativa, datos relevantes sobre procesos como la segmentación de las estructuras oculares (ver Figura 1); es el caso de Samper et al. (2020), que presentan una metodología para la detección y segmentación del disco óptico y los vasos sanguíneos. También se han propuesto trabajos e investigaciones que brindan información sobre el reconocimiento de las anomalías necesarias para poder establecer y dar un diagnóstico sobre enfermedades que se pueden presentar en el globo ocular, un ejemplo de esto es la investigación de Gondal et al. (2018), en donde hacen uso de una red neuronal convolucional (CNN, por sus siglas en inglés) para realizar una discriminación de las regiones oculares en el ojo con ayuda de múltiples imágenes provenientes de una base de datos que contiene imágenes de retinas en diferentes estados, para posteriormente analizarlas y detectar las lesiones que puedan estar presentes en ellas.

Por último, también se han propuesto trabajos para indicar la importancia de factores que son determinantes al momento de lograr un diagnostico automatizado de enfermedades oculares, en especial la retinopatía diabética por medio de métodos de DL. Es el caso de Moreira et al. (2020), aquí se presenta el desarrollo de redes tipo CNN para detectar retinopatía diabética junto con un apartado descriptivo sobre el impacto de la resolución de las imágenes y la red en la precisión de la predicción.

El DL ofrece algunas ventajas como el procesamiento de gran cantidad de imágenes con el uso de unidades de procesamiento gráfico (GPU, por sus siglas en inglés) y unidades de 
procesamiento tensorial (TPU, por sus siglas en inglés). Gracias a todas estas características las técnicas de DL han podido superar los métodos tradicionales en varias tareas de análisis de imágenes y visión por computadora. Este éxito ha motivado su aplicación al análisis de imágenes médicas, incluidas, por supuesto, las imágenes oftalmológicas. (Perdomo \& González, 2019).

Este artículo presenta una revisión del panorama actual de las técnicas y modelos de CNN aplicados a las imágenes oculares retinianas para lograr un diagnóstico automatizado de la retinopatía diabética a partir de la identificación de signos de lesiones ocasionadas por esta enfermedad, como lo son los micro aneurismas (MAs), exudados (EXs), hemorragias y anomalías microvasculares intrarretinianas (IRMA, por sus siglas en inglés).

Se muestran los diferentes conjuntos de datos de imágenes oculares de acceso público más populares en el ámbito científico. Adicionalmente, se hace una breve explicación de los enfoques utilizados por diferentes investigadores y científicos junto con sus respectivos resultados.

Todo esto con el objetivo de lograr una revisión útil para las investigaciones futuras de las personas que deseen seguir la línea de estudio de la detección de la RD con ayuda de las técnicas de DL.

La organización de este documento es como sigue: en la Sección 2 se presentan las generalidades de la detección automatizada de la RD, incluyendo su definición, los principales síntomas y anomalías que ella empieza a desarrollar en el ojo humano, sus etapas, los diferentes grados que puede presentar y el marco general que se debe seguir para su detección.

En la Sección 3 se muestran los diferentes conjuntos de datos de imágenes oculares de acceso público más populares y utilizados por los diferentes trabajos académicos presentes en el estado del arte. Posterior a esto, en la Sección 4 se exponen las métricas de rendimiento más comunes usadas por los investigadores para hacer la comparativa de sus resultados con base a otros trabajos previamente desarrollados.

En la Sección 5 se plantean los diferentes métodos e investigaciones recientes, basados en

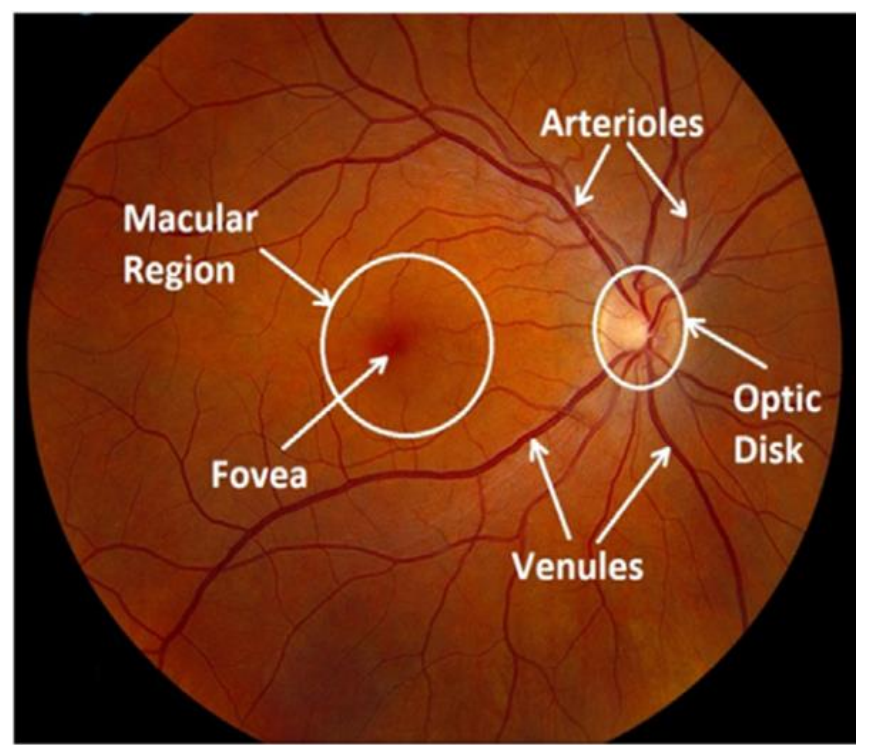

Figura 1. Imagen de fondo de ojo con la región macular centrada.

Fuente: Tomado de (Badar et al., 2020)

técnicas de DL aplicadas a la detección automatizada de la RD. En la Sección 6 se presenta un apartado de discusión de acuerdo con la opinión presentada por los autores.

Finalmente, en la Sección 7 se encuentran las conclusiones derivadas de este artículo de revisión del estado del arte.

\section{Detección automática de la retinopatía diabética}

\subsection{Retinopatía Diabética}

La retinopatía sigue siendo una de las complicaciones microvasculares más debilitantes de la diabetes y la principal causa de ceguera adquirida que afecta aproximadamente a 4,2 millones de personas en todo el mundo, con proyecciones de hasta 191 millones de personas afectadas para 2030 a nivel global. (Drankowska et al., 2019)

Además de ser una complicación de la diabetes que afecta los ojos, es una enfermedad progresiva que se diagnostica de acuerdo con algunas anormalidades clínicas de difícil detección, debido a que es asintomática hasta altas etapas del desarrollo de la enfermedad, y si no se realiza un tratamiento adecuado evoluciona en una patología mayor conocida como edema macular diabético que podría conllevar a la pérdida irreversible de la 
visión. (Giancardo et al., 2011, 2012; Rudas \& Torres, 2013)

Según Mayo Clinic (2018) cualquier persona con diabetes tipo 1 o 2 puede padecer este trastorno. Cuanto más tiempo lleve la persona con diabetes y cuanto menos se haya controlado el azúcar en la sangre, mayor es la probabilidad de presentar esta complicación en los ojos. Esta afirmación es soportada por Ishtiaq et al. (2020) cuando explica la forma en la que se desarrolla la retinopatía diabética en el ojo humano, esto es como sigue, la sangre es transportada a las capas del tejido retiniano por micro vasos sanguíneos que requieren un nivel de azúcar en sangre mantenido y un flujo sanguíneo sin obstrucciones. Si una gran cantidad de azúcares, como glucosa o fructosa, se acumulan en la sangre, estos vasos sanguíneos comienzan a descomponerse debido a la distribución inadecuada de oxígeno a las células. Las anomalías estructurales (ver Figura 2) ocurren como resultado de la disminución de la tasa metabólica que conduce a la RD.

Los principales síntomas y patologías de la RD incluyen:

Microaneurismas: son deformaciones en las paredes de los vasos sanguíneos. (Mookiah et al., 2013). Son difíciles de detectar con la oftalmoscopia convencional si no se tiene experiencia, pero aparecen como pequeñas manchas rojas con limites nítidos. (Jimenez, 2014)
Neovascularización: también llamada isquemia retiniana, ocurre cuando el cierre de los vasos sanguíneos retinianos genera que el suministro de oxígeno, que llega a las regiones retinianas, sea limitado. (Kowluru \& Chan, 2008)

El crecimiento del endotelio vascular intenta compensar la disminución del suministro de oxígeno, lo que da como resultado la formación de pequeños vasos sanguíneos débiles en la superficie de la retina, lo que conduce a la pérdida de la visión. (Badar et al., 2020)

Hemorragias: son producto del colapso de los microaneurismas y son visualmente apreciables como manchas de sangre sobre la superficie de la retina, de tamaño muy superior a las de los microaneurismas. (Rudas \& Torres, 2013)

Exudados duros: son objetos de color amarillo brillante o blanco en la retina. Estos objetos tienen una apariencia cerosa y bordes afilados contra el fondo de los vasos sanguíneos. Los exudados duros se desarrollan debido a la pérdida de sangre de las venas y los exudados tienen forma circular alrededor de los vasos. (Salamat et al., 2019)

Exudados suaves: también llamados manchas de algodón, son infartos de la capa de fibras nerviosas; tienen un aspecto más blanquecino. (Mittal \& Rajam, 2020

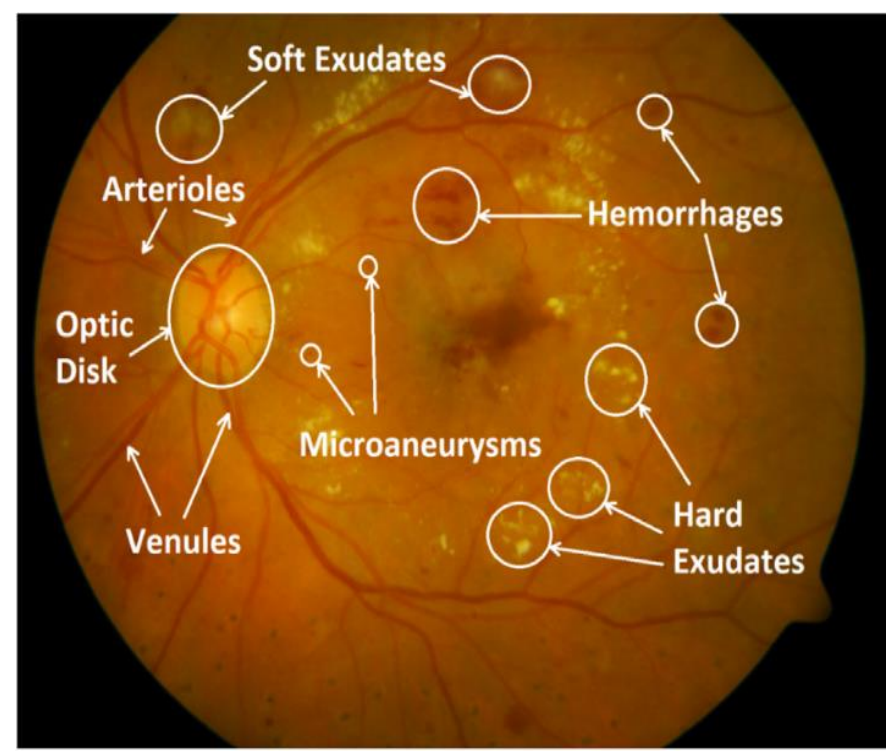

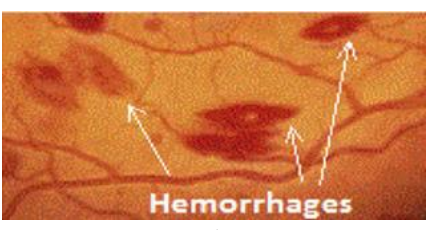

a)

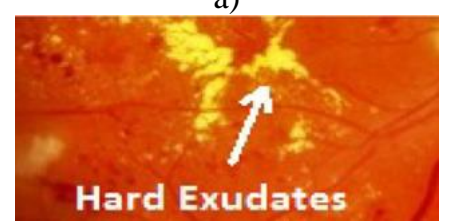

b)

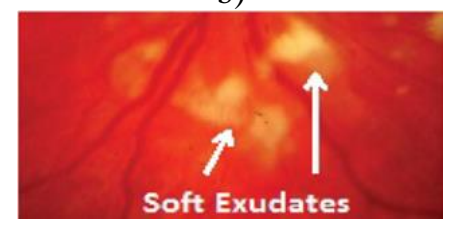

c)

Figura 2. (a) Imagen de la retina con sus patologías. (b) Hemorragias. (c) Exudados duros. (d) Exudados suaves.

Fuente: Tomado de (Badar et al., 2020) 


\subsection{Etapas de la retinopatía diabética}

La RD se divide en dos etapas principales: no proliferativa y proliferativa, llamada así por la ausencia o presencia de nuevos vasos sanguíneos anormales que emanan de la retina. (Fraser et al., 2017). Según Mittal \& Rajam (2020), la primera etapa, la no proliferativa (NPDR, por sus siglas en inglés) se subdivide en las siguientes fases: (1) fase medio, (2) fase moderada y (3) fase severa (ver Figura 3). De acuerdo con Randive et al. (2019), la NPDR crece con el daño de los vasos sanguíneos dentro de la retina, debido a que hace que la retina se hinche y se humedezca más, debido a la fuga de líquidos en las regiones de esta. La segunda etapa, considerada la etapa avanzada, es la retinopatía diabética proliferativa (PDR, por sus siglas en inglés).

Esta etapa, de acuerdo con la investigación realizada por Asiri et al. (2019), se caracteriza por una proliferación vascular anormal dentro de la retina hacia la cavidad vítrea. Estos frágiles vasos sanguíneos nuevos pueden sangrar en la cavidad vítrea y causar una pérdida visual severa debido a una hemorragia vítrea.

Cabe resaltar que la mayoría de los pacientes con retinopatía diabética, en sus etapas tempranas, no

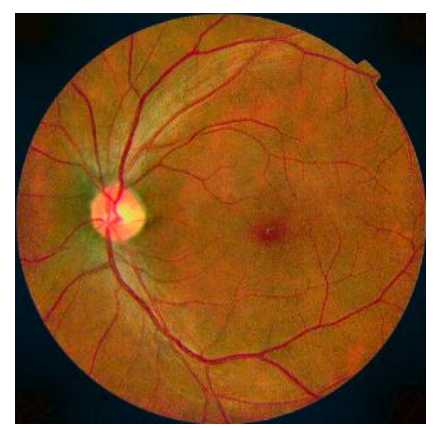

a)

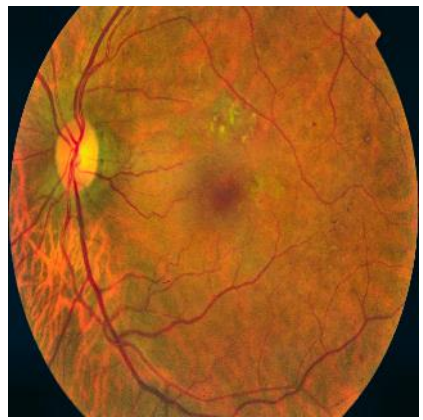

b)

notan muchos cambios en la calidad de su visión. Es por eso por lo que una persona con diabetes debe realizarse constantes chequeos médicos para así lograr un oportuno diagnóstico de la enfermedad y comenzar un tratamiento efectivo ya que cuando se presentan síntomas como visión borrosa $\mathrm{o}$ deficiencia en la percepción de colores significa que ya la enfermedad ha ido evolucionando considerablemente.

\subsection{Grados de la retinopatía diabética}

La clasificación del grado de la RD es una actividad de suma importancia en el proceso de detección y diagnóstico de las enfermedades de la retina (Asiri et al., 2019).

Se cuenta con varios sistemas que se encargan de brindar un estándar para la calificación de los cambios de tipo vascular, que se presentan y pueden observarse en las imágenes de fondo de ojo ocasionados por la $\mathrm{RD}$, como la clasificación que fue implementada gracias al Estudio de tratamiento temprano de la retinopatía diabética (ETDRS, por sus siglas en inglés) y el protocolo de clasificación dado por el Ministerio de Salud Neozelandés (Ministry of Health, 2016), en el cual según Asiri et al. (2019) solo se toma un campo por ojo, que se centra en la fóvea.

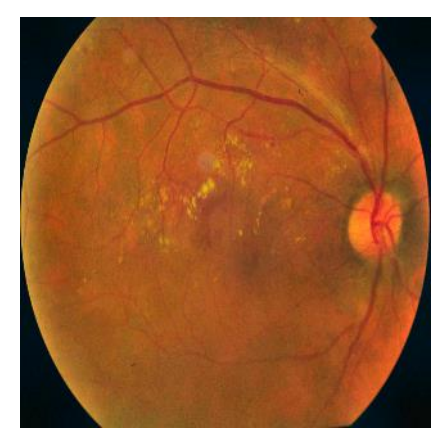

c)

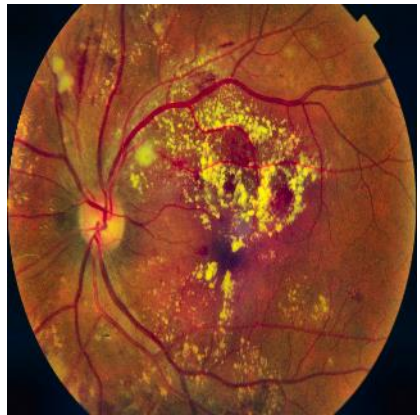

d)

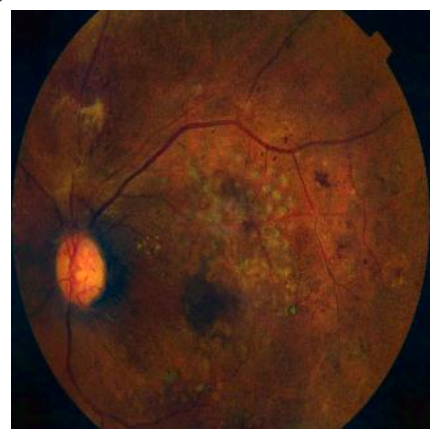

e)

Figura 3. Imágenes típicas de fondo de retina: (a) Normal, (b) Fase media NPRD, (c) Fase moderada NPRD, (d) Fase severa NPRD, (e) Retinopatía diabética proliferativa.

Fuente: Tomadas de conjunto de imágenes APTOS 2019 Blindness Detection. 
El protocolo de clasificación antes mencionado se ve representado y detallado en la Tabla 1.

\subsection{Marco general para los procesos de detección de la RD}

El marco general para la detección, segmentación y clasificación implica los pasos específicos de preprocesamiento, extracción/selección de características, elección de un método de clasificación adecuado y, finalmente, evaluación de los resultados. Los sistemas de clasificación DR se pueden dividir en dos tipos según el procedimiento de aprendizaje: aprendizaje supervisado y no supervisado. (Asiri et al., 2019)

Los modelos de aprendizaje supervisado son aquellos en los que se aprenden funciones, relaciones que asocian entradas con salidas, por lo que se ajustan a un conjunto de ejemplos de los que conocemos la relación entre la entrada y la salida deseada. Este hecho, incluso, llega a proporcionar una de las clasificaciones más habituales en el tipo de algoritmos que se desarrollan, así, dependiendo del tipo de salida, suele darse una subcategoría que diferencia entre modelos de clasificación, si la salida es un valor categórico (por ejemplo, una enumeración, o un conjunto finito de clases), y modelos de regresión, si la salida es un valor de un espacio continuo, es decir, el sistema se enseña utilizando datos etiquetados para inferir el mapeo funcional. (Asiri et al., 2019; Li et al., 2016; Sancho Caparrini, 2020)

Por otra parte, los modelos de aprendizaje no supervisado son aquellos en los que no estamos interesados en ajustar pares (entrada, salida), sino en aumentar el conocimiento estructural de los datos disponibles (y posibles datos futuros que provengan del mismo fenómeno). (Sancho Caparrini, 2020) Es decir, este tipo de modelo hace que la red aprenda por sí misma secuencias o patrones ocultos a partir de las similitudes que encuentra en todo el conjunto de imágenes del que se alimenta. (Arunkumar \& Karthigaikumar, 2017).

Tabla 1. Clasificación de grados de retinopatía diabética.

\begin{tabular}{|c|c|c|}
\hline Grado & Signos clínicos & Decisión \\
\hline R0: No RD & No existen anormalidades. & $\begin{array}{l}\text { Tipo1: Volver a examinar a los } 2 \text { años, } \\
\text { ajustando los modificadores clínicos. } \\
\text { Tipo 2: Reevaluación a los } 2-3 \text { años, } \\
\text { ajustando los modificadores clínicos. La } \\
\text { presencia de modificadores clínicos } \\
\text { puede requerir una reevaluación más } \\
\text { temprana. }\end{array}$ \\
\hline R1: Leve & Menos de 5 microaneurismas o puntos de hemorragia & $\begin{array}{l}\text { Reevaluación a los } 2 \text { años dependiendo } \\
\text { de los modificadores clínicos. }\end{array}$ \\
\hline R2: Media & $\begin{array}{l}\text { - Más de } 4 \text { microaneurismas o puntos de hemorragia. } \\
\text { - Exudados > } 2 \text { diámetros de disco de la fóvea. } \\
\text { - Algunas manchas y hemorragias más grandes son } \\
\text { aceptables. Si hay más de 20MA o hemorragias por } \\
\text { campo fotográfico se debe actualizar a R3 Moderado. }\end{array}$ & Volver a evaluar después de 12 meses. \\
\hline R3: Moderado & $\begin{array}{l}\text { Cualquier característica de R2. } \\
\text { Manchas y hemorragias más grandes. } \\
\text { Hasta un cuadrante de reborde venoso }\end{array}$ & Volver a evaluar después de 6 meses. \\
\hline R4: Severa & $\begin{array}{l}\text { Uno o más de: } \\
\text { - Anomalías microvasculares intrarretinianas definidas } \\
\text { (IRMA). } \\
\text { - Dos cuadrantes o más de cuentas cenosas. } \\
\text { - Cuatros cuadrantes de manchas o hemorragias más } \\
\text { grandes. }\end{array}$ & Revisión por oftalmólogo en 6 semanas. \\
\hline R5: Proliferativa & $\begin{array}{l}\text { Uno o más de: } \\
\text { - Neovascularización } \\
\text { - Hemorragia subhialoidea o vítrea. } \\
\text { - Desprendimiento de retina por tracción o gliosis de } \\
\text { retina. }\end{array}$ & $\begin{array}{l}\text { Remisión urgente a un oftalmólogo; } \\
\text { considere revisar dentro de } 2 \text { semanas. }\end{array}$ \\
\hline
\end{tabular}

Fuente: Adaptado de (Ministry of Health, 2016) 
Un ejemplo del modelo de una estructura de CNN aplicada a las imágenes de fondo de ojo se puede observar en la Figura 4.

\section{Conjuntos de datos de imágenes oculares (Datasets)}

En esta sección se mostrará un benchmarking de los conjuntos de datos de mayor uso en las investigaciones $\mathrm{y}$ trabajos relacionados a la detección de la RD por medio de técnicas de DL, junto con una breve descripción característica de cada una de las bases de imágenes oculares que se mencionarán.

ROC (Retinopathy Online Challenge): contiene 100 imágenes digitales a color de fondo de ojo con microaneurismas en todas las imágenes. Estas imágenes se encuentran aleatoriamente distribuidas y se dividen en 50 imágenes para entrenamiento de la red y 50 imágenes para pruebas. (Niemeijer et al., 2010) El enlace para acceder a esta base de datos de imágenes es http://webeye.ophth.uiowa.edu/ROC/

E-Ophta: este conjunto de datos se divide en 2 categorías: una llamada E-Ophta MA y la otra EOphta EX. E-Ophta MA posee 148 imágenes con microaneurismas y pequeñas hemorragias, y 233 imágenes sin lesiones; en cambio E-Ophta EX posee un conjunto de 47 imágenes de fondo de ojo con una segmentación de exudados y 35 imágenes sin ningún tipo de lesión y que se encuentran etiquetadas como imágenes normales. (E. Decencière et al., 2013) El enlace para acceder a este dataset es: http://www.adcis.net/en/thirdparty/e-ophtha/
Kaggle DR Dataset: esta base de datos es proporcionada por EyePACS, (n.d.) para colaborar en la investigación y desarrollo de trabajos relacionados a la detección y diagnóstico de la RD. En total posee 88,702 imágenes, de las cuales 35,126 son para tareas de entrenamiento y 53,576 son para tareas de pruebas. El enlace para acceder a este dataset es: https://www.kaggle.com/c/diabeticretinopathy-detection/data

DIARETDB0: consiste en 130 imágenes de fondo de ojo, de las cuales 110 presentan signos de RD y 20 son de ojos sanos. En esta base de datos las imágenes que pertenecen al grupo de RD contienen la etiqueta de los signos que presentan, es decir, exudados, microaneurismas, hemorragias $y$ neovascularización. (Tomi Kauppi et al., n.d.) El enlace para acceder a ella es: https://www.it.lut.fi/project/imageret/diaretdb0/

DIARETDB1: la base de datos consta de 89 imágenes de fondo de ojo en color, de las cuales 84 contienen al menos signos no proliferativos leves (microaneurismas) de la retinopatía diabética, y 5 se consideran normales: que no contienen ningún signo de la retinopatía diabética según todos los expertos que participaron en la evaluación. Las imágenes se capturaron utilizando una cámara de fondo de ojo digital con campo de visión de 50 grados con diferentes configuraciones de imagen. Los datos corresponden a una situación práctica buena (no necesariamente típica), donde las imágenes son comparables y pueden usarse para evaluar el desempeño general de los métodos de diagnóstico. (T Kauppi et al., n.d.) El enlace para ir a esta base de datos es: https://www.it.lut.fi/project/imageret/diaretdb1/

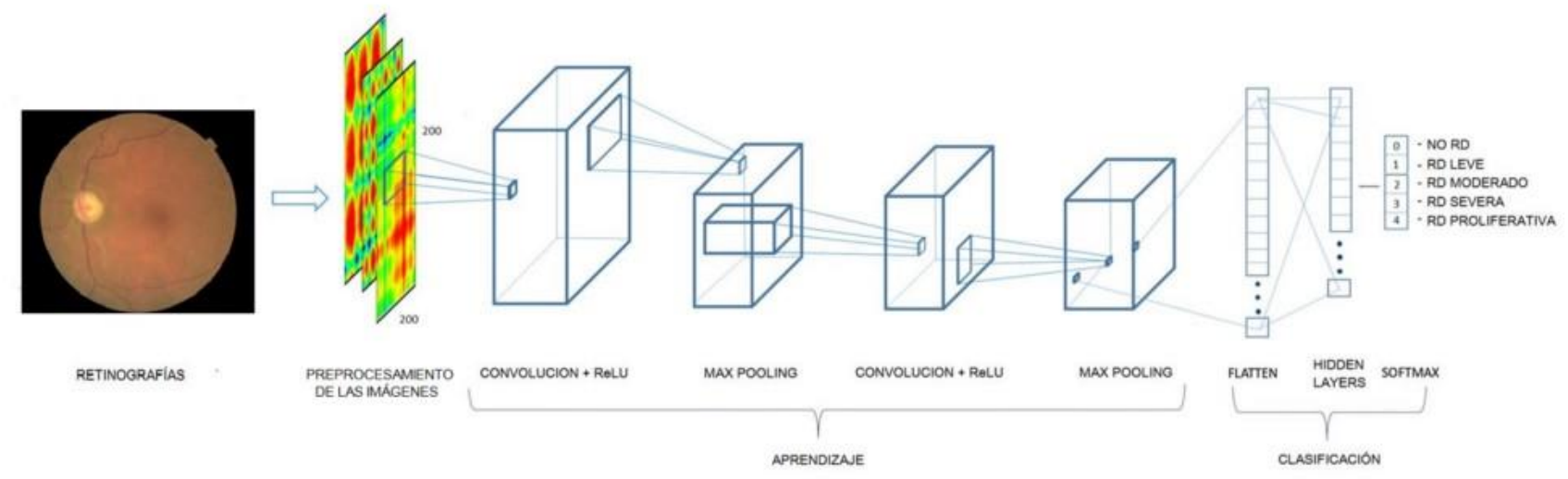

Figura 4. Modelo de CNN aplicado a las imágenes retinográficas.

Fuente: (Diagnóstico de Retinopatía Diabética Aplicando Redes Neuronales Convolucionales | by EAl| Data Science Bolivia | Medium, n.d.) 
Messidor: consta de 1.200 imágenes numéricas de color de fondo de ojo. Las imágenes se capturaron utilizando 8 bits por plano de color a $1440 * 960,2240 * 1488$ o $2304 * 1536$ píxeles. Posee 800 imágenes con dilatación pupilar (una gota de tropicamida al $0,5 \%$ ) y 400 sin dilatación. Todo el conjunto de imágenes se encuentra empaquetado en 3 juegos. Cada conjunto está dividido en 4 subconjuntos comprimidos que contienen 100 imágenes en formato TIFF y un archivo Excel con diagnósticos médicos para cada imagen. (Etienne Decencière et al., 2014). El enlace de esta base de datos es el siguiente: http://www.adcis.net/en/thirdparty/messidor/

DRIVE: la base de datos DRIVE se ha establecido para permitir estudios comparativos sobre la segmentación de los vasos sanguíneos en las imágenes de la retina. Consta de 40 fotografías, de las cuales 33 no presentan ningún tipo de RD y 7 muestran signos de RD temprana leve. La resolución de las imágenes es de $768 * 584$ píxeles. En este conjunto de datos 20 imágenes son de entrenamiento y la misma cantidad para pruebas. (Aggarwal \& Khare, 2015). El enlace para acceder a ella es: https://drive.grand-challenge.org/DRIVE/

STARE: consta de 400 imágenes. Estas poseen anotaciones de expertos según las características visibles de cada imagen, las cuales se encuentran tabuladas en archivos de texto. El enlace para acceder a esta base de datos es el siguiente: https://cecas.clemson.edu/ ahoover/stare/

\section{Métricas de rendimiento}

A continuación, se mostrarán las métricas de rendimiento más utilizadas por los investigadores, para poder establecer una comparativa entre los resultados obtenidos por los trabajos académicos realizados por otras personas o por ellos mismos en el diagnóstico de las enfermedades oculares, de los que disponen en su estado del arte y los resultados obtenidos en su actual investigación. Para hacer esta comparativa se utilizan lo que conocemos como métricas estadísticas, estas son las que van a mostrar qué tan en acuerdo o en desacuerdo están el experto y el método propuesto para calificar una enfermedad ocular. (Farsiu et al., 2014; Perdomo \& González, 2019)
Estas métricas de rendimiento son: (ver Tabla 2). Donde:

TP = Positivos verdaderos (número de píxeles del vaso correctamente clasificados).

$\mathrm{TN}=$ Negativo verdadero (número de píxeles de fondo correctamente clasificados)

FP = Falsos positivos (número de píxeles de fondo clasificados incorrectamente).

$\mathrm{FN}=$ Falsos negativos (número de píxeles del vaso incorrectamente clasificados).

$p_{o}=$ Probabilidad de acuerdo o clasificación correcta entre los calificadores.

$p_{e}=$ Probabilidad de acuerdo de azar entre los calificadores.

\section{Extracción de características y métodos de DL como herramienta para el diagnóstico automatizado de la $\mathrm{RD}$}

De acuerdo a la investigación ejecutada por Randive et al. (2019), para clasificar la RD, cada imagen del fondo digital está representada por un vector de características visuales llamado Firma. Se encuentra que los microaneurismas, las hemorragias, los exudados y las anomalidades microvasculares intraretinianas aparecen con varios vectores significativos de características visuales o Firmas como son el tamaño, la forma, el color, la intensidad, la textura, la densidad de vasos sanguíneos, el contraste, entre otros.

Es por esto por lo que muchos investigadores centran el desarrollo de su estudio en la extracción de dichas características, ya que así se puede determinar si existe algún tipo de anomalía, teniendo como referencia una imagen de fondo de ojo en estado sano. Es el caso de Arunkumar \& Karthigaikumar, (2017) el cual presenta un trabajo para el diagnóstico de enfermedades oculares a través de la extracción de características de DL y un clasificador SVM (Suppor Vector Machine) de varias clases. Chetoui \& Akhloufi (2020) propuso un método con el cual, gracias a la extracción de características, logró demostrar que el modelo era capaz de identificar de manera eficiente diferentes signos de RD. La red propuesta logró unas métricas de rendimiento considerablemente altas, con un AUC de 0,986 usando la base de datos EyePACS. 
Tabla 2. Métricas de rendimiento.

\begin{tabular}{|c|c|c|}
\hline Medida & Formula & Descripción \\
\hline Sensitividad (Se o recall) & $\frac{T P}{T P+F N}$ & $\begin{array}{l}\text { Relación entre los verdaderos positivos clasificados y los } \\
\text { verdaderos positivos reales en la verdad básica. También } \\
\text { conocido como tasa de verdaderos positivos (TPR). }\end{array}$ \\
\hline Especificidad (Sp) & $\frac{T N}{(T N+F P)}$ & $\begin{array}{l}\text { Relación entre los verdaderos negativos clasificados y los } \\
\text { verdaderos negativos reales en la verdad básica. (1-SP) también } \\
\text { se conoce como tasa de falsos positivos (FPR). }\end{array}$ \\
\hline Exactitud (Acc) & $\frac{T P+T N}{T P+T N+F P+F N}$ & $\begin{array}{l}\text { La precisión mide la proporción de píxeles clasificados } \\
\text { correctamente (tanto de los vasos como de los no vasos) con } \\
\text { respecto al número total de píxeles en el campo de visión de la } \\
\text { imagen (FOV). }\end{array}$ \\
\hline Precisión (Pr) & $\frac{T P}{(T P+F P)}$ & El porcentaje de casos positivos detectados. \\
\hline Área bajo la curva (AUC) & $\frac{S e+S p}{2}$ & $\begin{array}{l}\text { Área cubierta por la curva ROC cuando se logra la } \\
\text { optimización. }\end{array}$ \\
\hline Puntuación -F & $2 * \frac{S e+P r}{(S e+P r)}$ & $\begin{array}{l}\text { F-score es el promedio ponderado de precisión y recuerdo. } \\
\text { Tiene en cuenta tanto los falsos positivos como los falsos } \\
\text { negativos. }\end{array}$ \\
\hline Coeficiente Kappa & $\frac{p_{o}-p_{e}}{1-p_{e}}$ & $\begin{array}{l}\text { El coeficiente Kappa refleja la concordancia interobservador, } \\
\text { puede tomar valores entre }-1 \mathrm{y}+1 \text {. Mientras más cercano a }+1 \text {, } \\
\text { mayor es el grado de concordancia interobservador, por el } \\
\text { contrario, mientras más cercano a }-1 \text {, mayor es el grado de } \\
\text { discordancia interobservador. (Cerda \& Villarroel Del, 2008) }\end{array}$ \\
\hline
\end{tabular}

Fuente: Tomado y adaptado de (Badar et al., 2020; Mittal \& Rajam, 2020; Perdomo \& González, 2019)

Y para MESSIDOR, MESSIDOR-2, DIARETDB0, DIARETDB1, STARE, IDRID, Eophtha y UoA-DR, logró un AUC de 0.963, 0.979, $0.986, \quad 0.988, \quad 0.964,0.957,0.984$ y 0.990 , respectivamente.

Por otra parte, Wu et al. (2016) propuso un método que utiliza una red neuronal convolucional profunda $(\mathrm{CNN})$ para poder conocer las características de apariencia del objetivo. Los resultados cualitativos y cuantitativos sobre los datos del fondo de retina demostraron que los autores lograron una precisión comparable con métodos más avanzados.

Zhao et al. (2018) presentan un algoritmo de segmentación y localización del disco óptico de forma automática, basado en una red tipo Faster RCNN y un conjunto de niveles con restricciones de forma. El algoritmo fue entrenado con 4.000 imágenes tomadas de Kaggle y probadas en la base de datos MESSIDOR, logrando una puntuación promedio de coincidencia del $85,4 \%$.

Maninis et al. (2016) expone en su investigación lo que se conoce como Comprensión profunda de la imagen retiniana (DRIU, por sus siglas en inglés), el cual es un marco unificado de análisis de imágenes retinianas que proporciona segmentación de los vasos retinianos y del disco óptico. Hace uso de $\mathrm{CNN}$, la cual en su arquitectura se encuentran dos conjuntos de capas especializadas para resolver la segmentación de los vasos retinianos y del disco óptico, así como el trabajo de (Qiaoliang Li et al., 2016) en donde presentan un método para la segmentación de dicha estructura ocular, pero haciendo uso de un método supervisado.

He et al. (2016) presenta una metodología en la que reformula explícitamente las capas de su red como funciones residuales de aprendizaje con 
referencia a las entradas de las capas, en lugar de aprender funciones no referenciadas.

Gondal et al. (2018) en su publicación muestra que en las imágenes de las retinas se detectan diferentes áreas de lesiones, fundamentales para el diagnóstico oportuno de la RD a nivel de imagen, con una alta precisión comparable, e incluso superando a los métodos supervisados. A nivel de las lesiones logró pocos falsos positivos con alta sensibilidad, sin embargo, la red está únicamente entrenada en etiquetas a nivel de imagen que no incluyen información sobre las lesiones existentes. Clasificando entre imágenes enfermas y sanas, Gondal et al. Logró un AUC de 0,954 usando la base de datos DiaretDB1.

Escorcia-Gutierrez et al. (2016) en su trabajo manifiesta que logró la segmentación de la principal estructura anatómica de la retina, la cual es el disco óptico, haciendo uso del algoritmo Convexity Shape Prior. Además, como aporte complementario explica la forma en que se alcanzó la extracción de los vasos sanguíneos por medio de filtros, como: el de onda media, mediana, Gaussiana y Gabor.

Zilly et al. (2015) propone un método basado en una CNN para la segmentación del disco y la copa óptica. Entrena un clasificador de regresión logística tipo softmax posterior al proceso de los filtros, los cuales aprenden a lo largo de varias capas y la salida de las capas anteriores alimentan a las capas siguientes.

Q Li et al. (2015) desarrolla una investigación enfocada a la aplicación de un método supervisado para la segmentación de los vasos de la retina. Esto lo hace con una red neuronal tipo CNN y usaron el conjunto de datos de DRIVE. Pero más tarde, Qiaoliang Li et al. (2016), propone una mejora al enfoque de segmentación de los vasos presentado anteriormente y expone una red neural amplia y profunda con gran capacidad para realizar el modelado de una transformación. Esta red, en lugar de analizar solo el píxel central, puede analizar y dar salida a un mapa de etiquetas de todos los píxeles para una imagen. Como característica adicional de este modelo presentado por Qiaoliang Li et al., se puede resaltar el hecho de que no necesita ningún paso de preprocesamiento de la imagen. En adición a esto, Mo \& Zhang, (2017), sigue la misma línea de segmentación de vasos y presenta una red totalmente convolucional supervisada en profundidad, aprovechando las características jerárquicas multinivel de las redes profundas. Usaron las bases de datos de DRIVE, STARE Y CHASE_DB1, logrando resultados exitosos.

Lim et al. (2016) describe una solución integral, para la segmentación del disco y la copa ópticos, basada en la aplicación de CNN. En esta solución hace uso de mapas de probabilidades, los cuales son refinados con conocimientos previos de las estructuras retinianas. Lim et al. hace énfasis en que logrando la proporción entre la copa y el disco óptico puede alcanzar una detección eficaz de enfermedades como el glaucoma y la RD. Este estudio lo hizo con ayuda de dos bases de datos muy populares, una de ellas MESSIDOR.

Abràmoff et al. (2016) hicieron una comparativa del rendimiento de un algoritmo mejorado de DL para la detección automatizada de la RD con el rendimiento publicado con anterioridad de ese mismo algoritmo. Para la clasificación usaron el estándar de referencia de consenso, reportado previamente de RD referible, definido como Clasificación Clínica Internacional de Retinopatía diabética moderada, no proliferativa severa (NPDR, por sus siglas en inglés), RD proliferativo y/o edema macular (ME, por sus siglas en inglés). Los resultados fueron los siguiente: $96,8 \%$ de sensibilidad, la especificidad fue del $87,0 \%$, con 6/784 de falsos negativos, lo cual significó un valor predictivo negativo del $99,0 \%$ y el AUC fue de 0,980 .

Saranya Rubini et al. (2019) proponen un modelo de aprendizaje profundo automatizado para la detección de la RD basado en CNN, la cual es capaz de analizar las imágenes de la retina y clasificarlas como sanas o defectuosas según los síntomas de la RD. El modelo posee cinco capas de convolución y cinco agrupadas, seguidas de una capa de abandono $\mathrm{y}$ tres capas completamente conectadas. Este modelo no necesita preprocesamiento y aprende características discriminatorias de alto nivel de los síntomas de DR a partir de las intensidades de píxeles para clasificar la imagen de la retina como sana o defectuosa. Se usaron los conjuntos de datos MESSIDOR y ROC, obteniéndose una precisión de detección del $97 \%$. 
Chetoui \& Akhloufi, (2020) desarrollaron un algoritmo de DL capaz de detectar signos de RD en imágenes del fondo de ojo de la retina. Utilizan los conjuntos de datos EyePACS, MESSIDOR, MESSIDOR-2, DIARETDB0, DIARETDB1, STARE, IDRID, E-ophtha y UoA-DR y más de 90.000 imágenes para evaluar la eficiencia del algoritmo. Además, implementaron un algoritmo de explicabilidad basado en el mapeo de activación de clases ponderado por gradiente para mostrar visualmente los signos seleccionados por el modelo, para clasificar las imágenes de la retina como positivos para RD. Los resultados obtenidos fueron: área bajo la curva (AUC) de 0,986, sensibilidad de 0,958 y especificidad de 0,971 para EyePACS. Para MESSIDOR, MESSIDOR-2, DIARETDB0, DIARETDB1, STARE, IDRID, E-ophtha y UoADR, el AUC es 0.963, 0.979, 0.986, 0.988, 0.964, $0.957,0.984$ y 0.990 , respectivamente.

Gangwar \& Ravi, (2021) exponen un trabajo en el que proponen un hibrido de DL para la detección automática de la RD. Este híbrido se compone de un bloque personalizado de capas CNN sobre el modelo de aprendizaje por transferencia de Inception-ResNet-v2 previamente entrenado. Usaron el conjunto de datos MESSIDOR y APTOS 2019 (conjunto de datos de Kaggle). Lograron una precisión de prueba del $72,33 \%$ y el $82,18 \%$ en el conjunto de datos MESSIDOR y APTOS, respectivamente.

Guisela \& Pozo, (2019) sugieren analizar e implementar modelos de redes neuronales artificiales supervisadas para la detección de estructuras vasculares retinianas de fondo de ojo. Aplicaron una red convolucional basada en la arquitectura U-Net, con ventanas de imagen de $16 \mathrm{x}$ 16 píxeles. La red convolucional estaba compuesta por cuatro capas convolucionales y tres capas deconvolucionales. Obtuvieron unos resultados de sensibilidad del $74,20 \%$, una especificidad del $90,70 \%$ y una exactitud del $88,22 \%$.

Merino Hernandez et al. (2019) desarrollaron un algoritmo innovador basado en métodos avanzados de clasificación (Deep Learning y Support Vector Machine) que identifica automáticamente las imágenes patológicas de forma eficaz y segura. El software discrimina automáticamente retinografías normales y patológicas. Los resultados obtenidos fueron sensibilidad de $94 \%$ y una especificidad del $96 \%$.

Auccahuasi et al. (2020) presentan una metodología para poder reconocer los exudados duros, que es la primera manifestación de la retinopatía diabética. Auccahuasi et al. describe una nueva alternativa para el reconocimiento automático de exudados duros en las imágenes de la retina. Por medio de una CNN se trabaja con un conjunto de datos compuesto por 906 imágenes de exudados duros y 1.068 imágenes que no corresponden a exudados duros, que se entrena con un grupo de 400 imágenes, con una prevalencia de 0,5 con respecto a la presencia de exudados duros.

Después de esto se hacen dos comprobaciones al clasificador, utilizando grupos de imágenes similares, como el grupo de pruebas y validación, obteniendo valores de sensibilidad de $0,92 \%$ en ambos casos y valores de especificidad de $0,92 \%$ y $0,93 \%$ para los grupos de imágenes de prueba y validación, respectivamente. Se realizaron mediciones adicionales para calcular el Valor Predictivo Positivo y el Valor Predictivo Negativo, obteniéndose valores de $0,9207 \%$ para el grupo de imágenes de prueba y $0,93 \%$ para el grupo de imágenes de validación. En el caso del Valor Predictivo Negativo se obtuvieron valores de 0,9292\% para el grupo de imágenes de prueba y 0,93\% para el grupo de imágenes de validación.

Moreira et al. (2020) formulan el desarrollo de CNNs para detectar la RD, y el impacto de la resolución y la red de imágenes en la precisión de la predicción. Obtiene unos resultados de un área de 0,93 bajo la curva característica operativa del receptor al aumentar la resolución de la arquitectura Inception-V3.

Samper et al. (2020) presentan una metodología para la detección y segmentación del disco óptico y los vasos sanguíneos en imágenes de fondo de ojo de pacientes con RD. Hace uso del conjunto de datos DRIVE y logra una precisión (Acc) de $95 \%$ y una especificidad (Sp) de $98 \%$ para la segmentación de los vasos sanguíneos y una precisión de 94,6\% y $97.8 \%$ en especificidad para la segmentación del disco óptico. 


\section{Discusión de la temática}

Los trabajos mostrados con anterioridad son muestra del extenso desarrollo e investigación que presenta en la actualidad la ciencia de la inteligencia artificial (IA) y su rama conocida como aprendizaje profundo (DL, por sus siglas en inglés) aplicada a la detección automatizada de la RD. Se observa que muchos son los investigadores que han logrado exitosos resultados en el proceso de segmentación de las estructuras oculares, detección de anomalías y patologías presentes en las imágenes de fondo de ojo, e incluso han logrado establecer algoritmos los cuales, de forma automática y con unas altas métricas de rendimiento, logran establecer el grado de RD que puede presentar un paciente, posterior a estos haber sido entrenados con diferentes conjuntos de datos de imágenes oculares mostrados en la Sección 3.

Pero el punto de discusión aquí es sobre la comparativa y la forma de interpretar los diferentes métodos expuestos. Como bien se expresó por los autores, los métodos y algoritmos desarrollados en los diferentes estudios e investigaciones fueron entrenados y probados usando conjuntos de datos diferentes, las métricas de rendimiento arrojan resultados con base en esos conjuntos de datos, y como bien se sabe, existen conjuntos de datos que poseen 30 - 40 imágenes, como es el caso de DRIVE, la cual cuenta con 40 imágenes, mientras que existen otros que poseen 400 y más imágenes, como Messidor, que posee 1.200. Además existen trabajos que no usan todas las métricas de rendimiento al momento de dar sus resultados, como por ejemplo: el caso de Abràmoff et al. (2016), el cual en sus resultados brinda la especificidad, la sensibilidad, el área bajo la curva y el valor predictivo negativo. Mientras que el trabajo realizado por Saranya Rubini et al. (2019) solo brinda el valor referente a la precisión en la detección. Todos estos son agentes que dificultan la comparativa de resultados, ya que se deben analizar muchos más factores para poder establecer cuál trabajo es considerablemente mejor que el otro en términos de eficiencia y eficacia, de acuerdo con los números arrojados por las métricas de rendimiento que brindan los autores en sus producciones.

\section{Conclusiones}

Las técnicas de Deep Learning han demostrado tener el potencial suficiente para desempeñar labores que requieran del análisis y procesamiento de imágenes, en este caso dirigido a un campo de la medicina como lo es el diagnóstico temprano de la enfermedad ocular conocida como Retinopatía Diabética en base a los diversos síntomas que se manifiestan a nivel de la retina. De la misma manera resulta muy viable la utilización de esta novedosa tecnología en otras áreas de trabajo, sin limitarse únicamente al campo médico, pudiendo adaptarse según el objeto de estudio que se tenga.

Aun así, por más completa que pueda parecer estar, el Deep Learning no ha sido explotado en su totalidad, siendo que existen innumerables posibilidades para su uso y que, con el correr de los años y a medida que se estudien las múltiples funcionalidades que puede brindar, surgirán modelos capaces de procesar situaciones de naturaleza más compleja, llegando a contribuir en gran medida a las labores de los profesionales en lo que a tiempo, costo y precisión se refiere.

En lo que respecta al impacto social que puede generar, es cuestión de familiarizar a los profesionales con este tipo de métodos, siendo muy importante darles a conocer los grandes beneficios que presentan los sistemas desarrollados, ya que, en el caso de la detección de una enfermedad tan perjudicial como lo es la Retinopatía Diabética, es muy importante el impacto que puede generar la implementación de dichos sistemas, permitiendo a más personas acceder a un diagnóstico confiable y más rápido por un costo más reducido, incremento considerable de la cantidad de pacientes diagnosticados de manera oportuna, lograr evitar numerosos casos de ceguera y, en consecuente, una mejoría en la calidad de vida de la población. 


\section{Referencias}

Abràmoff, M. D., Lou, Y., Erginay, A., Clarida, W., Amelon, R., Folk, J. C., \& Niemeijer, M. (2016). Improved Automated Detection of Diabetic Retinopathy on a Publicly Available Dataset Through Integration of Deep Learning. Investigative Ophthalmology \& Visual Science, 57(13), 5200-5206. https://doi.org/10.1167/iovs.16-19964

Aggarwal, M., \& Khare, V. (2015). Automatic localization and contour detection of Optic disc. https://doi.org/10.1109/ICSPCom.2015.715068 $\underline{6}$

Arunkumar, R., \& Karthigaikumar, P. (2017). Multi-retinal disease classification by reduced deep learning features. Neural Computing and Applications, 28(2), 329-334. https://doi.org/10.1007/s00521-015-2059-9

Asiri, N., Hussain, M., Al Adel, F., \& Alzaidi, N. (2019). Deep learning based computer-aided diagnosis systems for diabetic retinopathy: A survey. Artificial Intelligence in Medicine, 99(December 2018), 101701. https://doi.org/10.1016/j.artmed.2019.07.009

Auccahuasi, W., Flores, E., Sernaque, F., Cueva, J., Diaz, M., \& Oré, E. (2020). Recognition of hard exudates using Deep Learning. Procedia Computer Science, 167(2019), 2343-2353. https://doi.org/10.1016/j.procs.2020.03.287

Badar, M., Haris, M., \& Fatima, A. (2020). Application of deep learning for retinal image analysis: A review. Computer Science Review, 35 , 100203. https://doi.org/10.1016/j.cosrev.2019.100203

Cerda, J. L., \& Villarroel Del, L. P. (2008). Evaluación de la concordancia inter-observador en investigación pediátrica: Coeficiente de Kappa. Rev Chil Pediatr, 79 (1), 54-58.

Chetoui, M., \& Akhloufi, M. A. (2020). Explainable end-to-end deep learning for diabetic retinopathy detection across multiple datasets. Journal of Medical Imaging, 7(4), 1-25. https://doi.org/10.1117/1.JMI.7.4.044503
Decencière, E., Cazuguel, G., Zhang, X., Thibault, G., Klein, J. C., Meyer, F., Marcotegui, B., Quellec, G., Lamard, M., Danno, R., Elie, D., Massin, P., Viktor, Z., Erginay, A., Lä̈, B., \& Chabouis, A. (2013). TeleOphta: Machine learning and image processing methods for teleophthalmology. IRBM, 34(2), 196-203. https://doi.org/10.1016/j.irbm.2013.01.010

Decencière, Etienne, Zhang, X., Cazuguel, G., Lay, B., Cochener, B., Trone, C., Gain, P., Ordonez, R., Massin, P., Erginay, A., Charton, B., \& Klein, J.-C. (2014). Feedback on a publicly distributed database: the Messidor database. Image Analysis \& Stereology, 33(3), 231-234. https://doi.org/10.5566/ias.1155

Diagnóstico de Retinopatía Diabética Aplicando Redes Neuronales Convolucionales | by EAl | Data Science Bolivia|Medium. (n.d.). Retrieved October 15, 2020, from https://medium.com/data-sciencebolivia/diagnóstico-de-retinopatía-diabéticaaplicando-redes-neuronales-convolucionales$\underline{8 d c 8328 c 3 e c 3}$

Drankowska, J., Kos, M., Kościuk, A., Marzęda, P., Boguszewska-Czubara, A., Tylus, M., \& ŚwięchZubilewicz, A. (2019). MMP targeting in the battle for vision: Recent developments and future prospects in the treatment of diabetic retinopathy. Life Sciences, 229, 149-156. https://doi.org/https://doi.org/10.1016/j.lfs.2019 .05 .038

Escorcia-Gutierrez, J., Torrents-Barrena, J., Romero-Aroca, P., Valls, A., \& Puig, D. (2016). Interactive optic disk segmentation ia discrete convexity shape knowledge using high-order functionals. Frontiers in Artificial Intelligence and Applications, 288, 39-44. https://doi.org/10.3233/978-1-61499-696-5-39

EyePACS. (n.d.). EyePACS. Retrieved October 4, 2020, from http://www.eyepacs.com/solution

Farsiu, S., Chiu, S. J., O'Connell, R. V., Folgar, F. A., Yuan, E., Izatt, J. A., \& Toth, C. A. (2014). Quantitative classification of eyes with and without intermediate age-related macular degeneration using optical coherence tomography. Ophthalmology, 121(1), 162-172. https://doi.org/10.1016/j.ophtha.2013.07.013 
Fraser, C., D'Amico, D., Nathan, D., Trobe, J., \& Mulder, J. (2017). Retinopatía diabética: clasificación y características clínicas UpToDate.

https://www.uptodate.com/contents/diabeticretinopathy-classification-and-clinical-features

Gangwar, A. K., \& Ravi, V. (2021). Diabetic Retinopathy Detection Using Transfer Learning and Deep Learning (pp. 679-689). Springer, Singapore. https://doi.org/10.1007/978-981-15$\underline{5788-064}$

Giancardo, L., Meriaudeau, F., Karnowski, T. P., Li, Y., Garg, S., Tobin, K. W., \& Chaum, E. (2012). Exudate-based diabetic macular edema detection in fundus images using publicly available datasets. Medical Image Analysis, 16(1), 216-226. https://doi.org/https://doi.org/10.1016/j.media.2 $\underline{011.07 .004}$

Giancardo, L., Meriaudeau, F., Karnowski, T. P., Yi, L., Tobin, K., Chaum, E., Giancardo, L., Meriaudeau, (2) F, Karnowski, TP, Li, (2) Y, Tobin, (3) $K W$ (2), \& Chaum, E. (2011). AUTOMATIC RETINA EXUDATES SEGMENTATION WITHOUT A MANUALLY LABELLED TRAINING SET. International Symposium on Biomedical Imaging. http://risa.uthsc.edu/dmed.

Gondal, W. M., Kohler, J. M., Grzeszick, R., Fink, G. A., \& Hirsch, M. (2018). Weakly-supervised localization of diabetic retinopathy lesions in retinal fundus images. Proceedings International Conference on Image Processing, ICIP, 2017-Septe, 2069-2073. https://doi.org/10.1109/ICIP.2017.8296646

Guisela, E., \& Pozo, R. (2019). ANÁLISIS DE CLASIFICADORES SUPERVISADOS PARA DETECTAR LA ESTRUCTURA VASCULAR EN IMÁGENES RETINIANAS PATOLÓGICAS. ESCUELA POLITÉCNICA NACIONAL.

He, K., Zhang, X., Ren, S., \& Sun, J. (2016). Deep residual learning for image recognition. Proceedings of the IEEE Computer Society Conference on Computer Vision and Pattern
Recognition,

2016-Decem, 770-778. https://doi.org/10.1109/CVPR.2016.90

Ishtiaq, U., Kareem, S. A., Rahayu, E., \& Faizal, M. (2020). Diabetic retinopathy detection through artificial intelligent techniques : a review and open issues. 15209-15252.

Jimenez, E. (2014, August 21). Retinografia digital $y$ https:/les.slideshare.net/jimenezcuadra/retinogr afia-digital-y-f-o

Kauppi, T, Kalesnykiene, V., Kamarainen, J.-K., Lensu, L., Sorri, I., Raninen, A., Voutilainen, R., Pietilä, J., Kälviäinen, H., \& Uusitalo, H. (n.d.). the DIARETDBI diabetic retinopathy database and evaluation protocol. https://doi.org/10.5244/C.21.15

Kauppi, Tomi, Kalesnykiene, V., Kamarainen, J.-K., Lensu, L., Sorri, I., Uusitalo, H., Kälviäinen, H., \& Pietilä, J. (n.d.). DIARETDBO: Evaluation Database and Methodology for Diabetic Retinopathy Algorithms.

Kowluru, R. A., \& Chan, P.-S. (2008). Capillary Dropout in Diabetic Retinopathy. In Diabetic Retinopathy (pp. 265-282). Humana Press. https://doi.org/10.1007/978-1-59745-563-311

Li, Q, Xie, L., Zhang, Q., Qi, S., Liang, P., Zhang, H., \& Wang, T. (2015). A supervised method using convolutional neural networks for retinal vessel delineation. 2015 8th International Congress on Image and Signal Processing (CISP), 418-422. https://doi.org/10.1109/CISP.2015.7407916

Li, Qiaoliang, Feng, B., Xie, L., Liang, P., Zhang, H., \& Wang, T. (2016). A cross-modality learning approach for vessel segmentation in retinal images. IEEE Transactions on Medical Imaging, 35(1), 109-118. https://doi.org/10.1109/TMI.2015.2457891

Lim, G., Cheng, Y., Hsu, W., \& Lee, M. L. (2016). Integrated optic disc and cup segmentation with deep learning. Proceedings - International Conference on Tools with Artificial Intelligence, ICTAI, 2016-Jапиа, 162-169. https://doi.org/10.1109/ICTAI.2015.36 
Maninis, K.-K., Pont-Tuset, J., Arbeláez, P., \& Van Gool, L. (2016). Deep Retinal Image Understanding. In S. Ourselin, L. Joskowicz, M. R. Sabuncu, G. Unal, \& W. Wells (Eds.), Medical Image Computing and Computer-Assisted Intervention -- MICCAI 2016 (pp. 140-148). Springer International Publishing.

Mayo Clinic. (2018). Retinopatía diabética Síntomas y causas. https://www.mayoclinic.org/es-es/diseasesconditions/diabetic-retinopathy/symptomscauses/syc-20371611

Merino Hernandez, M., Jauregui Garcia, M., Agirrezabala Iturralde, J., Urruzola Lizarribar, A., Guibelalde Gonzalez, A., Prat Madrazo, M., Vergara Mitxeltorena, I., \& Soto Ruiz de Gordoa, M. (2019). Desarrollo de un software de cribado automático de retinopatía diabética. International Journal of Integrated Care, 19(4), 74. https://doi.org/10.5334/ijic.s3074

Ministry of Health. (2016). Diabetic Retinal Screening, Grading, Monitoring and Referral Guidance.

Mittal, K., \& Rajam, V. M. A. (2020). Computerized retinal image analysis - a survey. In Multimedia Tools and Applications (Vol. 79, Issues 31-32). Multimedia Tools and Applications. https://doi.org/10.1007/s11042-020-09041-y

Mo, J., \& Zhang, L. (2017). Multi-level deep supervised networks for retinal vessel segmentation. International Journal of Computer Assisted Radiology and Surgery, 12(12), 2181-2193. https://doi.org/10.1007/s11548-017-1619-0

Mohammadi, F., Esmaeili, M., Javadzadeh, A., Tabar, H. A., \& Rasta, S. H. (2019). The computer based method to diabetic retinopathy assessment in retinal images: A review. Electronic Journal of General Medicine, 16(2), 1-13. https://doi.org/10.29333/ejgm/108619

Mookiah, M. R. K., Acharya, U. R., Chua, C. K., Lim, C. M., Ng, E. Y. K., \& Laude, A. (2013). Computer-aided diagnosis of diabetic retinopathy: A review. Computers in Biology and
Medicine, 43(12), 2136-2155. https://doi.org/https://doi.org/10.1016/j.compbi omed.2013.10.007

Moreira, F., Schaan, B., Schneiders, J., Reis, M., Serpa, M., \& Navaux, P. (2020). Impacto da Resolução na Detecção de Retinopatia Diabética com uso de Deep Learning. 494-499. https://doi.org/10.5753/sbcas.2020.11546

Niemeijer, M., Van Ginneken, B., Cree, M. J., Mizutani, A., Quellec, G., Sanchez, C. I., Zhang, B., Hornero, R., Lamard, M., Muramatsu, C., Wu, X., Cazuguel, G., You, J., Mayo, A., Li, Q., Hatanaka, Y., Cochener, B., Roux, C., Karray, F., ... Abramoff, M. D. (2010). Retinopathy online challenge: Automatic detection of microaneurysms in digital color fundus photographs. IEEE Transactions on Medical Imaging, 29(1), 185-195. https://doi.org/10.1109/TMI.2009.2033909

Perdomo, O. J., \& González, F. A. (2019). A Systematic Review of Deep Learning Methods Applied to Ocular Images. Ciencia e Ingeniería Neogranadina, 30(1), 9-26. https://doi.org/10.18359/rcin.4242

Randive, S. N., Senapati, R. K., \& Rahulkar, A. D. (2019). A review on computer-aided recent developments for automatic detection of diabetic retinopathy. Journal of Medical Engineering and Technology, 43(2), 87-99. https://doi.org/10.1080/03091902.2019.157679 $\underline{O}$

Rodríguez, F. P. (2019). Prototipo de redes neuronales para predecir posibles complicaciones por diabetes [Universidad Autónoma de Ciudad Juárez]. https://doi.org/10.1017/CBO9781107415324.00 $\underline{4}$

Rudas, J., \& Torres, G. S. (2013). Detección de patologías derivadas de las afecciones diabéticas: una revisión del análisis digital de imágenes de retina The detection of pathologies from diabetic disease : a review of digital retinal image analysis. Ingeniería y Desarrollo. Universidad Del Norte., 3461. 
Salamat, N., Missen, M. M. S., \& Rashid, A. (2019). Diabetic retinopathy techniques in retinal images: A review. Artificial Intelligence in Medicine, 97(January 2018), 168-188. https://doi.org/10.1016/j.artmed.2018.10.009

Samper, N. M., Escorcia-gutierrez, J., Acosta, M. G., \& Torres, M. (2020). Metodología de Segmentación de la Estructura Ocular en Imágenes de Fondo de Ojo de Pacientes con Retinopatía Diabética Methodology of Segmentation the Main Retinal Anatomical Structures in Fundus Images from Patients with Diabetic Retinopathy. Prospectiva, 18. https://doi.org/http:://doi.org/10.15665/rp.v18i2 .2401

Sancho Caparrini, F. (2020). Aprendizaje Supervisado y No Supervisado. http://www.cs.us.es/ fsancho/?e $=77$

Saranya Rubini, S., Saai Nithil, R., Kunthavai, A., \& Sharma, A. (2019). Deep convolutional neural network-based diabetic retinopathy detection in digital fundus images. Advances in Intelligent Systems and Computing, 900, 201-209. https://doi.org/10.1007/978-981-13-3600-3_19

Scanlon, P. H. (2019). Diabetic retinopathy. Medicine (United Kingdom), 47(2), 77-85. https://doi.org/10.1016/j.mpmed.2018.11.013

Wu, A., Xu, Z., Gao, M., Buty, M., \& Mollura, D. J. (2016). Deep vessel tracking: A generalized probabilistic approach via deep learning. 2016 IEEE 13th International Symposium on Biomedical Imaging (ISBI), 1363-1367. https://doi.org/10.1109/ISBI.2016.7493520

Zhao, H., Chen, X., Zhang, D., Zhu, W., \& Shi, F. (2018). Automatic localization and segmentation of optical disk based on faster $R$-CNN and level set in fundus image. In E. D. Angelini \& B. A. Landman (Eds.), Medical Imaging 2018: Image Processing (Vol. 10574, p. 65). SPIE. https://doi.org/10.1117/12.2292950

Zilly, J. G., Buhmann, J. M., \& Mahapatra, D. (2015). Boosting convolutional filters with entropy sampling for optic cup and disc image segmentation from fundus images. In Lecture Notes in Computer Science (including subseries
Lecture Notes in Artificial Intelligence and Lecture Notes in Bioinformatics) (Vol. 9352). Springer Verlag. https://doi.org/10.1007/978-3319-24888-2_17 